\title{
Polynomial Division using Dynamic Arrays, Heaps, and Packed Exponent Vectors *
}

\author{
Michael Monagan and Roman Pearce \\ Department of Mathematics, Simon Fraser University \\ Burnaby, B.C. V5A 1S6, CANADA. \\ mmonagan@cecm.sfu.ca and rpearcea@cecm.sfu.ca
}

\begin{abstract}
A common way of implementing multivariate polynomial multiplication and division is to represent polynomials as linked lists of terms sorted in a term ordering and to use repeated merging. This results in poor performance on large sparse polynomials.

In this paper we use an auxiliary heap of pointers to reduce the number of monomial comparisons in the worst case while keeping the overall storage linear. We give two variations. In the first, the size of the heap is bounded by the number of terms in the quotient(s). In the second, which is new, the size is bounded by the number of terms in the divisor(s).

We use dynamic arrays of terms rather than linked lists to reduce storage allocations and indirect memory references. We pack monomials in the array to reduce storage and to speed up monomial comparisons. We give a new packing for the graded reverse lexicographical ordering.

We have implemented the heap algorithms in $\mathrm{C}$ with an interface to Maple. For comparison we have also implemented Yan's "geobuckets" data structure. Our timings demonstrate that heaps of pointers are comparable in speed with geobuckets but use significantly less storage.
\end{abstract}

\section{Introduction}

In this paper we present and compare algorithms and data structures for polynomial division in the ring $P=F\left[x_{1}, x_{2}, \ldots, x_{n}\right]$ where $F$ is a field. We are interested in (i) exact division of $f \in P$ by a single polynomial $g \in P$, that is testing whether $g \mid f$ and if so, computing the quotient $q=f / g$, (ii) exact division of $f \in P$ by a polynomial $g \in P$ modulo a triangular set of polynomials in $F\left[x_{i}, x_{i+1}, \ldots, x_{n}\right]$, and (iii) computing the remainder of $f \in P$ divided by a set of polynomials $\left\{g_{1}, g_{2}, \ldots, g_{s}\right\} \in P$. Since many algorithms in computer algebra use modular methods for efficiency, that is, they compute modulo primes, we will want to divide over characteristic $p$ as well as characteristic 0 .

We consider distributed polynomial representations that sort the terms of the polynomial with respect to a monomial ordering. See (3) or (4) for background material on monomial orderings. The orderings that we are most interested in

\footnotetext{
* This work was supported by NSERC of Canada and the MITACS NCE of Canada
} 
are the pure lexicographical ordering (lex), the graded lexicographical ordering (grlex), and the graded reverse lexicographical ordering (grevlex). In the grlex ordering one first sorts the terms by total degree and then by lexicographical order. For example, the polynomial

$$
-9 x^{4}-7 x^{3} y z+6 x^{2} y^{3} z+8 y^{2} z^{3}
$$

when written with terms in descending grlex order with $x>y>z$ is

$$
6 x^{2} y^{3} z-7 x^{3} y z+8 y^{2} z^{3}-9 x^{4} .
$$

The data structure used to represent polynomials will have a direct impact on the efficiency of the division algorithm. The data structure used by the Axiom system (7) for Gröbner basis computations is the SDMP (Sparse Distributed Multivariate Polynomial) data structure. This is a linked list of terms where each term is a pair $(c, e)$, where $c$ is a (pointer to) a coefficient and $e$ is a pointer to the exponent vector, which is an array of machine integers. Using $\langle a, b, c, \ldots\rangle$ to denote an array, $[a, b, c, \ldots]$ to denote a linked list, and $(c, e)$ to denote a pair of pointers, the polynomial above would be represented as

$$
[(6,\langle 2,3,1\rangle),(-7,\langle 3,1,1\rangle),(8,\langle 0,2,3\rangle),(-9,\langle 4,0,0\rangle)] .
$$

Recall the division algorithm. Following the notation of Cox, Little, and O'Shea (3), we let $L T(f), L M(f)$, and $L C(f)$ denote the leading term, the leading monomial, and the leading coefficient of a polynomial $f$, respectively. These depend on the term ordering but satisfy $L T(f)=L C(f) L M(f)$.

\section{The Division Algorithm.}

Input: $f, g_{1}, g_{2}, \ldots, g_{s} \in F\left[x_{1}, \ldots, x_{n}\right], F$ a field.

Output: $q_{1}, q_{2}, \ldots, q_{s}, r \in F\left[x_{1}, \ldots x_{n}\right]$ satisfying $f=q_{1} g_{1}+q_{2} g_{2}+\ldots+q_{s} g_{s}+r$.

1: Set $\left(q_{1}, q_{2}, \ldots, q_{s}\right):=(0,0, \ldots, 0)$.

2: Set $p:=f$.

3: While $p \neq 0$ do

4: Find the first $g_{i}$ s.t. $\operatorname{LM}\left(g_{i}\right) \mid L M(p)$.

5: If no such $g_{i}$ exists then set $r:=r+L T(p)$ and $p:=p-L T(p)$

6: else set $\left(q_{i}, p\right):=\left(q_{i}+t, p-t \times g_{i}\right)$ where $t=L T(p) / L T\left(g_{i}\right)$.

7: Output $\left(q_{1}, q_{2}, \ldots, q_{s}, r\right)$.

Remark: If one wishes to test if $\left(g_{1}, \ldots, g_{s}\right) \mid f$ with 0 remainder then Step 5 should be modified to stop execution and output false.

If polynomials are represented as linked lists of terms sorted in descending order in the term ordering then accessing the leading term $L T(f)$ takes constant time, the operation $p-L T(p)$ (link to the remaining terms of $p$ ) is constant time and $r+L T(p)$ can be done in constant time by maintaining a pointer to the last term of $r$. The most expensive step is the subtraction $p-t \times g_{i}$. This requires a "merge" - one simultaneously walks down the linked list of terms in $p$ and the linked list of terms in $g_{i}$ comparing monomials. In the worst case the merge must walk to the end of both $p$ and $g_{i}$. 


\subsection{Storage management and non-local memory references.}

We identify two sources of inefficiency in the division algorithm when the SDMP data structure is used. The first is the many intermediate pieces of storage that need to be allocated when we multiply $t g_{i}$, for example, storage for new exponent vectors in $t g_{i}$. The second is the memory references that occur during the merge when we walk down the linked lists and, for each term, link to the exponent vectors to compare monomials. These memory references cause a loss in efficiency when the polynomials are too large to fit inside the computer's cache. On a 2.4 GHz AMD Opteron 150 with $400 \mathrm{MHz}$ RAM we measured the loss of speed at a factor of 6 .

These two problems can be eliminated by representing polynomials as arrays with the coefficients and exponents stored in place. For example, $6 x^{2} y^{3} z-7 x^{3} y z+$ $8 y^{2} z^{3}-9 x^{4}$ could be stored as

$$
\langle 6,2,3,1,-7,3,1,1,8,0,2,3,-9,4,0,0\rangle .
$$

The difference $p-t g_{i}$ can be computed efficiently by merging with two arrays: one, $p$, that we are copying terms out of, and another, $p^{\prime}$, that we are forming the difference $p-t \times g_{i}$ inside. When the merge is complete we interchange the roles of $p$ and $p^{\prime}$ for the next iteration of the division algorithm. If $p^{\prime}$ is too small to store all of the terms of $p$ and $-t \times g_{i}$ we allocate a new $p^{\prime}$ with $50 \%$ more terms than are needed to reduce the chance of another allocation in the future.

But, there is a loss of efficiency; instead of copying pointers (one word) we must now copy exponent vectors ( $n$ words). This loss can be reduced by packing multiple exponents into each word. For example, Macaulay (5) uses dynamic arrays and packed exponent vectors. Macaulay identifies the monomials $1, z, y, x, z^{2}, z y, y^{2}, z x, y x, x^{2}, \ldots$ with non-negative integers $0,1,2,3, \ldots$ to encode each monomial as an integer. The polynomial $6 x^{2} y^{3} z-7 x^{3} y z+8 y^{2} z^{3}-9 x^{4}$ would be represented as an array of 8 words

$$
\langle+6,63,-7,49,+8,36,-9,33\rangle .
$$

This encoding gives a very compact representation with fast monomial comparisons, but monomial multiplication and division are slow. In (1), Bachmann and Schönemann compare different monomial packings including the Macaulay encoding. They show that packing exponent vectors produces a modest speedup (a factor of 1.5 to 2) for Gröbner basis computations modulo a machine prime with the SDMP data structure. They also show that simpler packing schemes are more efficient overall than the Macaulay encoding.

\subsection{The problem of too many monomial comparisons.}

When using merging to subtract $p-t g_{i}$, a serious inefficiency may occur when $\# p$, the number of terms in $p$, is much larger than \# $g_{i}$, the number of terms in a divisor $g_{i}$. Consider $g=(x+1), q=y^{n}+\ldots+y^{2}+y$ and let $p=g q=$ $x y^{n}+\ldots+x+y^{n}+\ldots+y$. If we compute $p$ by adding $x q$ to $q$ using merging, the 
merge does $n$ comparisons which is efficient. In dividing $f$ by $g$ the first quotient is $y^{n}$ and we subtract $y^{n} g=x y^{n}+y^{n}$ from $p=x y^{n}+\ldots+x y+y^{n}+\ldots+y$. The merge does $n$ comparisons to find $y^{n}$ in $p$. The full division does $n$ such merges so the total number of comparisons is $O\left(n^{2}\right)$, much worse than multiplication.

One solution is to represent the polynomial $p$ as a binary search tree. Then $\mathrm{LT}(p)$ can be computed with $O(\log \# p)$ monomial comparisons and the difference $p-t g_{i}$ can be computed with $O\left(\# g_{i} \log \# p\right)$ comparisons. However binary trees suffer from the same cache performance problems as linked lists.

A very nice solution is the "geobucket" data structure of Yan (12), which is used by the Singular (6) computer algebra system and others. Geobuckets are described in detail in Section 2. In the geobucket data structure a polynomial $p$ with \#p terms is represented by an array of $O(\log \# p)$ "buckets" where the $i$ 'th bucket $p_{i}$ is a linked list of at most $2^{i}$ terms. To subtract $t \times g_{i}$ from $p$ one subtracts $t \times g_{i}$ from the $i^{\prime}$ th bucket of $p$ where $2^{i-1}<\# g_{i} \leq 2^{i}$. Subtraction is done by merging two linked lists. The idea is that asymptotic efficiency is not lost when we merge linked lists with a similar number of terms, e.g., their length differs by at most a factor of two.

In this paper we use an auxiliary "heap of pointers" instead. When dividing $p$ by $\left\{g_{1}, g_{2}, \ldots, g_{s}\right\}$ we maintain a heap of pairs with quotient terms and pointers back into the divisors $\left\{g_{1}, g_{2}, \ldots, g_{s}\right\}$. The pointers indicate which terms have yet to be multiplied and subtracted from $p$.

Suppose we are dividing $f$ by $g$. Let $f=g q+r$ where $q$ is the quotient and $r$ the remainder. With geobuckets, division does $O(\# g \# q(\log \# g+\log \# q))$ comparisons (12). If we use a heap, division does $O(\# g \# q \log \# q)$ comparisons. A second key advantage of using a heap is that it requires only $O(\# q)$ space, and, if we need to compute the remainder, $O(\# r)$ space to write down the remainder. By comparison, the simple merge and geobucket algorithms may require $O(\# g \# q+\# r)$ space. The main disadvantage of using a heap is that for dense polynomials the merge and geobucket algorithms are better; they do only $O(\# g \# q)$ comparisons. A third advantage of using a heap is that we delay all coefficient arithmetic until we need to do it. This can result in significant speedups when we want to test if $g$ divides $f$ but $g$ does not divide $f$.

The idea of using a heap for sparse polynomial arithmetic was first investigated by Johnson in 1974 (8). Heaps were used in Altran (2), one of the earliest computer algebra systems. We are not aware of any other computer algebra system that has used heaps for polynomial arithmetic despite their good asymptotic performance. Heaps were not considered by Stoutemyer in (11) which, as far as we are aware, is the only systematic experiment ever done comparing different polynomial data structures on a computer algebra system's test suite.

\subsection{Organization of the Paper}

In Section 2 we describe how we encode and pack monomials for different term orderings. Our packing for graded reverse lexicographical order is new. In Section 3 we give the main algorithms that use heaps of pointers. Two algorithms are presented. The first algorithm bounds the size of the heap by the number of terms 
in the quotients $\left\{q_{1}, q_{2}, \ldots, q_{s}\right\}$. In the second algorithm, the size of the heap is bounded by the number of terms in the divisors $\left\{g_{1}, g_{2}, \ldots, g_{s}\right\}$. This algorithm is new, and it is particularly useful for polynomial GCD computations because the gcd $G$ of two polynomials $A$ and $B$ typically has fewer terms, often much fewer, than the quotients $A / G$ and $B / G$.

We have implemented the division algorithms in the $\mathrm{C}$ programming language. We create polynomials in Maple and call our $\mathrm{C}$ code from Maple using Maple's foreign function interface (see Ch. 8 of (10)). For comparison we have also implemented Yan's geobucket data structure using dynamic arrays with packed exponent vectors. Details of our geobucket implementation are given in Section 2. In Section 4 we give some benchmarks comparing the simple merging algorithm with Yan's geobucket representation and our heap algorithms, using packed and unpacked exponent vectors.

Our conclusions may be summarized as follows. Simple merging is not competitive with either heaps or geobuckets on sparse problems. The heap algorithms are as fast as geobuckets but use far less memory. Geobuckets do the fewest monomial comparisons, but heaps tend to be faster on large problems because they use cache more efficiently. For all algorithms, packing exponents significantly improves performance, especially on 64-bit machines.

\section{Dynamic Array Implementation}

Consider the minimum amount of work that a sparse algorithm must do. As noted by Johnson (8), a multiplication $f g$ must construct all $\# f \# g$ products of terms because the monomials generated may be distinct. These terms are merged to form the result. Similarly, to divide $f$ by $g$ we construct the quotient $q$ incrementally while subtracting $q g$ from $f$, merging $\# f+\# q(\# g-1)$ terms to do the division. Note, it is \#g-1 and not \#g because $-q \times L T(g)$ cancels terms so only $-q \times(g-L T(g))$ needs to be merged. Let $r=f-q g$. The number of monomial divisions attempted is $\# q+\# r$. To divide $f$ by $\left\{g_{1}, \ldots, g_{s}\right\}$ with quotients $\left\{q_{1}, \ldots, q_{s}\right\}$ we merge $\# f+\sum_{i=1}^{s} \# q_{i}\left(\# g_{i}-1\right)$ terms and attempt $\sum_{i=1}^{s}\left(\# q_{i}\right) i+(\# r) s$ monomial divisions if for each term we loop through the divisors in order.

Sorting the result imposes an additional cost in monomial comparisons if a function is called to compare terms with respect to an ordering. The $n m$ terms of a product can be naively sorted using $O(n m \log (n m))$ comparisons, but if the polynomials are sorted we can exploit that fact to do only $O(n m \log (\min (n, m)))$ comparisons. In either case the logarithmic factor is significant - it means that monomial comparisons dominate sparse polynomial computations when the cost of coefficient arithmetic is low.

\subsection{Packed Monomial Representations}

After an initial experiment we decided to base our monomial representations on Bachmann and Schönemann's scheme (1), which is used in Singular. The 
defining feature of this scheme is that a monomial stores two components: a (possibly weighted) total degree and a vector of exponents. An inline function compares the degree and the exponent vector in lexicographic order, and two global variables invert the results of these comparisons separately. To compare in reverse lexicographic order we reverse the variables and invert all the comparisons. Figure 1 shows the unpacked representations of $x^{2} y^{3} z^{4}$ with respect to four common orders with $x>y>z$. Shading is used to indicate where the results of comparisons are inverted.

Fig. 1. Unpacked $x^{2} y^{3} z^{4}$ with $x>y>z$.

\begin{tabular}{|c|c|c|}
\hline $\mathrm{x}$ & $\mathrm{y}$ & $\mathrm{z}$ \\
\hline 2 & 3 & 4 \\
\hline \multicolumn{3}{|c|}{ lexicographic order } \\
$\mathrm{z}$ & $\mathrm{y}$ & $\mathrm{x}$ \\
\hline 4 & 3 & 2 \\
\hline
\end{tabular}

reverse lexicographic order

\begin{tabular}{|c|c|c|c|}
\hline deg & $\mathrm{x}$ & $\mathrm{y}$ & $\mathrm{z}$ \\
\hline 9 & 2 & 3 & 4 \\
\hline \multicolumn{4}{|c|}{ graded lexicographic order } \\
deg & $\mathrm{z}$ & $\mathrm{y}$ & $\mathrm{x}$ \\
\hline 9 & 4 & 3 & 2 \\
\hline
\end{tabular}

graded reverse lexicographic order

Fig. 2. Packed $x^{2} y^{3} z^{4}$ with $x>y>z$.

\begin{tabular}{|l:c|c|c|}
$\mathrm{x}$ & $\mathrm{y}$ & $\mathrm{z}$ \\
\hline 2 & 3 & 4 & \\
\hline
\end{tabular}

lexicographic order

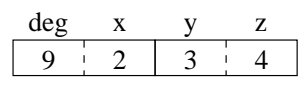

graded lexicographic order

\begin{tabular}{|c|c:c|c:|}
\hline weight & $\mathrm{x}$ & $\mathrm{y}$ & $\mathrm{z}$ \\
\hline$w$ & 2 & 3 & 4 \\
\hline
\end{tabular}

weighted lexicographic order

\begin{tabular}{|c:c|c:c|c:c|}
\multicolumn{1}{c}{ deg } & $\mathrm{x}+\mathrm{y}$ & $\mathrm{y}$ & $\mathrm{x}$ & $\mathrm{z}$ \\
\hline 9 & 5 & 3 & 2 & 4 & \\
\hline
\end{tabular}

graded reverse lexicographic order

\begin{tabular}{|c|l:l|l|l|}
\hline weight & $\mathrm{z}$ & $\mathrm{y}$ & $\mathrm{x}$ \\
\hline$w$ & 4 & 3 & 2 & $\vdots$ \\
\hline
\end{tabular}

weighted reverse lexicographic order

To pack monomials we use bitwise or and shift operations on machine words so that byte order is automatically taken into account. Our diagrams use bigendian format. We reserve the most significant bit of each exponent as a guard bit for monomial division. This operation subtracts machine words and uses a bitmask to detect if an exponent is negative. The mask also stores the length of the monomials which is needed by every routine. Weighted orders use the entire first word for the weighted degree since this can be large. We restrict the weights to non-negative integers so that the weighted degree is also a non-negative integer.

For graded orders we use the same number of bits for the total degree as for each exponent so that all monomials up to the maximum degree are encoded efficiently. Note that it is especially easy to determine an optimal packing for these orders using bounds on the total degree. If the polynomials are already sorted with respect to the order then we can examine their leading terms and repack the polynomials in linear time.

Figure 2 shows the packed representations of $x^{2} y^{3} z^{4}$ for five monomial orders with two exponents per machine word. Notice how monomial comparisons are reduced to lexicographic and reverse lexicographic comparisons of machine words. The encodings should all be straightforward except for graded reverse lexicographic order. In that case recall that the total degree only requires as 
many bits as a single packed exponent. The first word of the monomial, which must be compared lexicographically unlike the rest, would contain relatively little information if it only stored the total degree.

Our first idea was to pack more information into the first word to decide monomial comparisons. Observe that the matrices $A$ and $B$ in Figure 2.1 both describe graded reverse lexicographic order in four variables. Let $V$ be an exponent vector. Then $A V$ is encoded in the first $|V|$ words of the unpacked representation. The matrix $B$ is obtained from $A$ by adding the previous rows of $A$ to each row of $A$, eliminating all negative entries. Thus $B V$ contains only non-negative integers that are compared lexicographically. We pack as much of $B V$ as possible into the first word of the monomial.

Fig. 3. Matrix representations of graded reverse lexicographic (grevlex) order.

$$
A=\left[\begin{array}{rrrr}
1 & 1 & 1 & 1 \\
0 & 0 & 0 & -1 \\
0 & 0 & -1 & 0 \\
0 & -1 & 0 & 0
\end{array}\right] \quad B=\left[\begin{array}{llll}
1 & 1 & 1 & 1 \\
1 & 1 & 1 & 0 \\
1 & 1 & 0 & 0 \\
1 & 0 & 0 & 0
\end{array}\right]
$$

Fig. 4. Packed representations of $x^{1} y^{2} z^{3} t^{4} u^{5} v^{6} w^{7}$ in grevlex order with 4 exponents per word. The exponents for $w, v, u$, and $x$ are redundant. In the second representation, all monomial comparisons can be decided on the basis of the first seven exponents, after looking at only two words.

\begin{tabular}{|c|c|c|c|c|c|c|c|c|c|c|}
\hline deg & X...v & x...u & x..t & w & $\mathrm{v}$ & $\mathrm{u}$ & $\mathrm{t}$ & $\mathrm{z}$ & $\mathrm{y}$ & $\mathrm{x}$ \\
\hline 28 & 21 & 15 & 10 & 7 & 6 & 5 & 4 & 3 & 2 & 1 \\
\hline deg & X...v & x...u & x..t & $\mathrm{t}$ & $\mathrm{z}$ & $\mathrm{y}$ & $\mathrm{x}$ & W & $\mathrm{v}$ & $\mathrm{u}$ \\
\hline 28 & 21 & 15 & 10 & 4 & 3 & 2 & 1 & 7 & 6 & 5 \\
\hline
\end{tabular}

However, this does not actually fix the problem since now the second word of the monomial contains information that can be derived from the first. Refer to the top of Figure 4, where $w=7, v=6$ and $u=5$ are known from 28 -21 , $21-15$, and $15-10$. Thus the second word now provides only one exponent with new information, but we can easily fix this by moving all but the last exponent of the second word to the end of the monomial, as in the bottom of Figure 4 . Then for $n$ variables the first $n$ exponents encode all of the information necessary to decide monomial comparisons in grevlex order.

One might wonder why we do not simply encode the vector $B V$. The reason is that for monomial division one must unpack and decode quotients to check that they are valid. An example is given below. In fact, we tried this representation initially and found that while it was quite compact for grevlex order, weighted orders were inefficient and reverse lexicographic order could not be implemented. Eventually we decided to store all of the exponents explicitly, and Bachmann and Schönemann's scheme was the obvious choice. 
Example 1. Consider $x^{2}$ and $y^{3}$ in graded reverse lexicographic order with $x>$ $y>z$. The exponent vectors are $U=[3,0,0]$ and $V=[0,2,0]$ respectively, and the matrix $B$ is shown below. The difference $B U-B V$ is non-negative even though $U-V=[3,-2,0]$.

$$
B=\left[\begin{array}{lll}
1 & 1 & 1 \\
1 & 1 & 0 \\
1 & 0 & 0
\end{array}\right] \quad B U=\left[\begin{array}{l}
3 \\
3 \\
3
\end{array}\right] \quad B V=\left[\begin{array}{l}
2 \\
2 \\
0
\end{array}\right] \quad B U-B V=\left[\begin{array}{l}
1 \\
1 \\
3
\end{array}\right]
$$

\subsection{Iterated Merging with Dynamic Arrays}

The classical approach to polynomial arithmetic is an iterated merge. To multiply $f$ by $g$ we compute $\sum_{i=1}^{\# f} f_{i} g$ by adding each $f_{i} g$ to the previous partial sum using a merge. Similarly, to divide $f$ by $g$ we compute terms of the quotient $q$ while subtracting each $q_{i} g$ from an intermediate polynomial $p$, which is initially $f$.

Our first goal was to implement these algorithms while avoiding memory allocation. We use two global arrays or "merge buffers" $p$ and $p^{\prime}$ which grow dynamically, and all merging takes place from $p$ into $p^{\prime}$. If $p^{\prime}$ does not have sufficient storage to hold the objects being merged then it is enlarged. To amortize this cost we allocate a new $p^{\prime}$ with $50 \%$ more storage than required. To further amortize the cost of memory allocation we reuse $p$ and $p^{\prime}$ in the next call to an algorithm rather than free them each time.

Fig. 5. Division using two dynamic arrays. The fourth term of p produced the quotient term $y$, and we are beginning to merge the rest of $p$ (two terms) with $-y$ times the rest of $g$ (two terms). The buffer $p^{\prime}$ is large enough to store the result. Otherwise we would enlarge it to six terms.

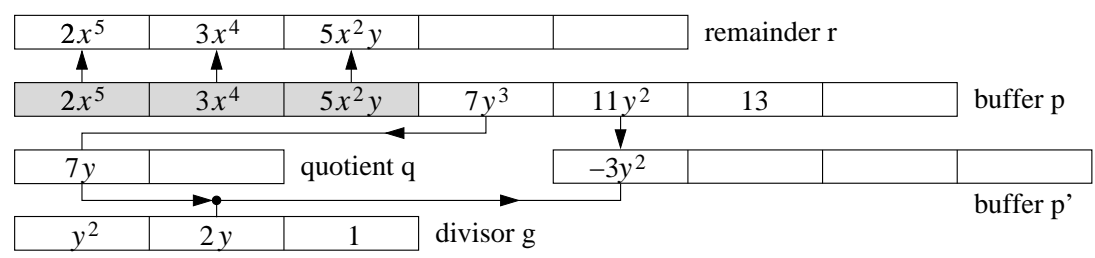

We describe our implementation of the division algorithm. To divide $f$ by $g$, we copy $f$ into $p$ and increment along the terms of $p$ until we reach the end or we find a term $p_{i}$ that is divisible by $L T(g)$. We copy the previous terms of $p$ to the remainder and if a reducible term was found, say $p_{i}=q_{j} L T(g)$, we merge the rest of $p$ with $-q_{j}(g-L T(g))$ into $p^{\prime}$, as shown in Figure 5 . The terms of $-q_{j}(g-L T(g))$ are constructed during the merge. Finally we interchange $p$ and $p^{\prime}$ by swapping pointers so that $p^{\prime}$ becomes $p$ for the next iteration of the algorithm and the storage for $p$ is recycled.

The complexity of this approach was analyzed by Johnson (8). He observed that for a multiplication $f g$ where $f$ has $n$ terms, $g$ has $m$ terms, and $f g$ has $n m$ 
terms, adding each $f_{i} g$ to the partial sum can require up to $i m-1$ monomial comparisons, making the total number of comparisons $\sum_{i=2}^{n} i m-n+1 \in O\left(n^{2} m\right)$. A similar result holds for division when the quotient has $n$ terms, the divisor has $m$ terms, and the dividend has $n m$ terms. Thus iterated merging can be very bad when the quotient is large.

It is interesting to note that $O\left(n^{2} m\right)$ comparisons may be required even if the product or dividend does not have $O(n m)$ terms, if terms introduced by the first $n / 2$ summands are canceled by the last $n / 2$ summands. We call this an intermediate blowup in the number of terms. One unfortunate feature of algorithms that add each $f_{i} g$ or $q_{i} g$ to a partial sum is that they allocate storage for all of these terms even when the end result is zero, as it will be for exact division. In Section 3 we will see that the heap algorithms avoid this problem by merging all of the partial products simultaneously.

For sparse polynomials an iterated merge uses about $2 \mathrm{~nm}$ terms of storage where $\mathrm{nm}$ is the size of the largest intermediate sum. If we always enlarge the buffers by $50 \%$ then we will use storage for about $3 \mathrm{~nm}$ terms on average. Quotient(s) and the remainder require additional storage if they are needed.

\subsection{Divide and Conquer Merging - Geobuckets}

A well-known alternative to iterated merging is divide-and-conquer merging, which is often used for polynomial multiplication. Let $f$ have $n$ terms and let $g$ have $m$ terms. If we compute $\sum_{i=1}^{n} f_{i} g$ by summing the first $n / 2$ and the last $n / 2$ summands recursively and adding their sums, then at most $C(n) \leq$ $2 C(n / 2)+n m-1 \in O(n m \log n)$ monomial comparisons are required. The method is efficient because it merges polynomials of similar size.

But how much memory is required? If each recursive call allocates memory for its own result then we can solve the same recurrence to find that $O(n m \log n)$ memory is needed. This is an order of magnitude larger than any possible result. Instead we could reuse a set of geometrically increasing buckets with $\{2 m, 4 m, \ldots, n m / 2\}$ terms for polynomials that we are waiting to merge, plus two arrays with $n m$ and $n m / 2$ terms for polynomials that we are currently merging. This simple "geobucket" algorithm is described below.

\section{Geobucket Multiplication.}

Input: $f=f_{1}+\cdots+f_{n}, g=g_{1}+\cdots+g_{m}$.

Output: $f g$.

1: Allocate buckets with $\left\{2 m, 4 m, \ldots, 2^{\left\lceil\log _{2}(n)\right\rceil-1} m\right\}$ terms.

2: Allocate dynamic arrays $p$ and $p^{\prime}$.

3: For $i:=1$ while $i \leq n$ do

4: Compute $f_{i} g$ and store it in $p$.

5: If $i<n$ merge $p$ and $f_{i+1} g$ into $p^{\prime}$ and swap $p$ and $p^{\prime}$.

6 : Set $i:=i+2$.

7: For $j:=1$ while bucket $[j] \neq 0$ do 8: Merge $p$ and bucket $[j]$ into $p^{\prime}$ and swap $p$ and $p^{\prime}$. 
9: Set bucket $[j]:=0$ and $j:=j+1$.

10: If $i \leq n$ set bucket $[j]:=p$ and $p:=0$.

11: For $j:=1$ to $2^{\left\lceil\log _{2}(n)\right\rceil-1}$ do

12: If bucket $[j] \neq 0$ merge $p$ and bucket $[j]$ into $p^{\prime}$ and swap $p$ and $p^{\prime}$.

13: Output $p$.

Thus $f_{1} g$ and $f_{2} g$ are merged and their sum is stored in bucket 1 , then $f_{3} g$ and $f_{4} g$ are merged and their sum is merged with $f_{1} g+f_{2} g$ and stored in bucket 2 , then $f_{5} g$ and $f_{6} g$ are merged and their sum is stored in bucket 1 , and so on, continuing in the manner of a depth-first search. If $n=2^{k}$ it is easy to see that $O(n m)$ storage is used. The buckets contain $(n-2) m$ terms, the array that stores the result will need $\mathrm{nm}$ terms, but the other array can have $\mathrm{nm} / 2$ terms. The total amount of storage required is $2.5 \mathrm{~nm}$ terms - only $50 \%$ more than for an iterated merge. If we always grow the arrays by an extra $50 \%$ then we can expect to allocate storage for about $3.25 \mathrm{~nm}$ terms in total.

Geobuckets were proposed by Yan (12) with three significant improvements. First, Yan's buckets have a small base and ratio that are independent of any problem to ensure good performance when objects of varying sizes are added to the geobucket. In the algorithm above the base is $2 m$ and the ratio is 2 , so objects with fewer than $m$ terms could be added more efficiently with a smaller bucket. Second, Yan always tries to store $p+$ bucket $[j]$ in bucket $[j]$ if possible to avoid creating bucket $[j+1]$. This decreases the amount of memory and increases the likelihood of combining terms on dense problems, resulting in fewer monomial comparisons. Finally, Yan describes a reasonably efficient scheme for coalescing the leading terms of the buckets to compute the leading term of the polynomial. This allows us to run the division algorithm with the intermediate polynomial $p$ stored as a geobucket. We state Yan's algorithm below for completeness.

\section{Geobucket Leading Term.}

Input: polynomial $f$ stored in bucket $[1 \ldots k]$.

Output: $L T(f)$ or FAIL when $f=0$, set bucket $[1 \ldots k]:=f-L T(f)$.

1 : Set $j:=0$, the bucket containing the leading term.

2: For $i:=1$ while $i \leq k$ do

3: If bucket $[i] \neq 0$ and $(j=0$ or $\operatorname{LM}($ bucket $[i])>\operatorname{LM}($ bucket $[j]))$ 4: Set $j:=i$

5: else if bucket $[i] \neq 0$ and $\operatorname{LM}($ bucket $[i])=\operatorname{LM}($ bucket $[j])$

6: Set $L C($ bucket $[j]):=L C($ bucket $[j])+L C($ bucket $[i])$.

7: Remove $L T$ (bucket $[i])$ from bucket $[i]$.

8: Set $i:=i+1$.

9: If $j=0$ then $f=0$ so output FAIL.

10: If $L C($ bucket $[j])=0$ remove this term from bucket $[j]$ and goto step 1 .

11: Set $t:=\operatorname{LT}($ bucket $[j])$.

12: Remove $L T$ (bucket $[j]$ ) from bucket $[j]$.

13: Output $t$. 
We implemented Yan's geobuckets using a single dynamic array so that its storage could be reused in subsequent calls. We chose a ratio of two because that is optimal for merging and our smallest bucket (the base) has four terms. We found that geobuckets performed very well, often using fewer monomial comparisons than expected.

For a sparse multiplication producing $n m$ terms geobuckets do $O(n m \log n)$ comparisons and store about $3.6 \mathrm{~nm}$ terms. This number can be derived as follows. The arrays (merge buffers) require $\mathrm{nm}$ and $\mathrm{nm} / 2$ terms, but we will allocate an extra $50 \%$ for each. The buckets have $n m$ terms, but the base (two) is independent of $m$ so we expect each bucket to be $75 \%$ full. The total is $4 n m / 3+(3 / 2)(n m+n m / 2)=(43 / 12) n m$ terms.

We can make a similar estimate for exact division when the dividend has $n m$ terms, however the complexity is $O(n m \log (n m))$ because of how leading terms are computed. The dividend is placed into the largest bucket, which we expect to be $75 \%$ full, so the storage for buckets is $2(4 \mathrm{~nm} / 3)=8 \mathrm{~nm} / 3$. Nothing is merged with the largest bucket since $\sum_{i=1}^{\# q} q_{i} g$ fits entirely in the smaller buckets, so the largest merge that we expect to do is to construct $\sum_{i=1}^{\# q / 2} q_{i} g$ which has $n m / 2$ terms. This requires arrays with $n m / 2$ and $n m / 4$ terms, plus the extra $50 \%$ that we allocate, bringing the total number of terms to $8 \mathrm{~nm} / 3+(3 / 2)(3 \mathrm{~nm} / 4)=$ $(91 / 24) n m$.

The actual amount of memory that geobuckets need for exact division tends to vary. It can be lower if the leading term computations frequently cancel terms in the buckets, reducing the size of the polynomials that are merged. For random sparse divisions we found that approximately $3.6 \mathrm{~nm}$ terms were used - about the same as for multiplication. The dynamic arrays were often the same size, about $3 \mathrm{~nm} / 5$ terms each.

\section{Heap Algorithms for Polynomial Arithmetic}

The heap algorithms are based on the following idea: rather than merge polynomials one by one into an intermediate object, we do a simultaneous $n$-ary merge using a heap. Consider the multiplication $f g$ where we merge $f_{i} g$ for $1 \leq i \leq \# f$. If we maintain a heap of $\# f$ pointers into $g$, sorted by the monomial of $f_{i} g_{j}$, we can repeatedly extract the largest $f_{i} g_{j}$ from the heap, merge it onto the end of the result, and insert its successor $f_{i} g_{j+1}$ into the heap if $j<\# g$. We illustrate this process in Figure 6 below.

The monomial of $f_{i} g_{j}$ is computed and stored in the heap when the term is inserted. It is used to determine the maximum element of the heap. This storage is reused for $f_{i} g_{j+1}$ so only $O(\# f)$ storage is required, in addition to storage for the result.

To divide $f$ by $g$ we merge the dividend $f$ with $-q_{i} g$ for each term $q_{i}$ of the quotient. The heap maintains a pointer into $f$ and we add a pointer into $-q_{i} g$ when $q_{i}$ is constructed. The algorithm extracts the largest term from the heap and continues to extract terms with an equal monomial, adding their coefficients to produce the next term of $f-\sum_{j=1}^{i} q_{j} g$. If this term is not zero we divide it by 
Fig. 6. Multiplication of $f=2 x^{4}+3 x^{3}+4 x$ and $g=x^{5}+5 x^{3}+7$ using a heap. The products $f_{1} g_{1}$ and $f_{2} g_{1}$ have been extracted and replaced by $f_{1} g_{2}$ and $f_{2} g_{2}$. We are now extracting $f_{1} g_{2}=10 x^{7}$ and writing it to the result. Its successor $f_{1} g_{3}=14 x^{4}$ will be inserted into the heap, and we will extract $f_{2} g_{2}$ and $f_{3} g_{1}$ to obtain $15 x^{6}+4 x^{6}=19 x^{6}$, the fourth term of the result.

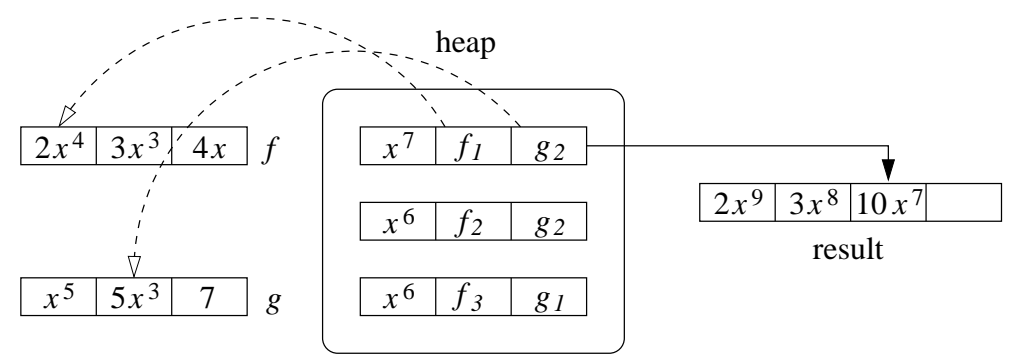

$L T(g)$ to obtain either a new term of the quotient $q_{i+1}$, or the next term of the remainder. When a quotient term is found we insert the second term of $-q_{i+1} g$ into the heap, increasing the size of the heap by one, along with the successors of the other terms that were extracted. There is no intermediate blowup in the number of terms that are stored - the maximum number of terms in the heap is \#q+1. We call this a "quotient heap" division.

The heap algorithms above were analyzed by Johnson (8) and used in Altran, one of the first computer algebra systems. For a binary heap of size $n$, inserting and extracting each term does $O(\log n)$ monomial comparisons. A multiplication that passes $n m$ terms through a heap of size $n$ does $O(n m \log n)$ comparisons the same as divide-and-conquer. Exact division $f \div g$ with $\# f=n m, \# g=m$, and the quotient $\# q=n$, passes $2 n m-n$ terms through a heap of size $n+1$, which is also $O(n m \log n)$ comparisons.

One problem with the heap algorithms is that they do $O(n m \log n)$ comparisons even when the polynomials are dense, whereas the simple merge and the divide-and-conquer algorithms do only $O(\mathrm{~nm})$ comparisons. In Section 3.2 we show how to modify the heap to make the heap algorithms efficient in the dense case as well.

Our main contribution is to modify the heap division algorithm to increment along the quotient(s) instead of the divisor(s). The resulting "divisor heap" algorithm does $O(n m \log m)$ comparisons and uses $O(m)$ storage, where $m$ is the size of the divisor(s). Our incentive comes from the gcd problem, where we compute $G=\operatorname{gcd}(A, B)$ and divide $A / G$ and $B / G$ to recover the cofactors. The divisor $G$ is typically small and the quotients (cofactors) are often big. The algorithm is also useful for computing over small towers of algebraic extensions, where the number of reductions usually exceeds the size of the extensions.

The modification is easy to do. The algorithm merges $f$ with $-g_{i} q$ for $2 \leq$ $i \leq \# g$ using a heap of size $\# g$, however we may merge $g_{i} q_{j-1}$ before $q_{j}$ is computed, in which case we can not insert the next term $g_{i} q_{j}$ into the heap because we can not compute its monomial. However, since $L T(g) q_{j}>g_{i} q_{j}$ for 
all $i>1$, we can safely wait for $q_{j}$ to be computed to insert the terms $g_{i} q_{j}$ with $i>1$ into the heap. We exploit the fact that the term $g_{i} q_{j}$ is greater than the term $g_{i+k} q_{j}$ for $k>0$, so if $\# q=j-1$ we encounter the strictly descending sequence $g_{2} q_{j}>g_{3} q_{j}>g_{4} q_{j}>\ldots$ in order. For each divisor $g$ we store an index $s$ of the largest $g_{s} q_{j}$ that is missing from the heap because $q_{j}$ is unknown. When a new term of the quotient is computed $(\# q=j)$ we compute all of the missing terms $\left\{g_{2} q_{j}, \ldots g_{s} q_{j}\right\}$ and insert them into the heap. Here we give the algorithm for one divisor.

\section{Divisor Heap Division.}

Input: $f, g \in F\left[x_{1}, \ldots, x_{n}\right], F$ a field, $g \neq 0$.

Output: $q, r \in F\left[x_{1}, \ldots x_{n}\right]$ with $f=q g+r$.

1 : If $f=0$ then output $(0, f)$.

2: Initialize $(q, r, s):=(0,0, \# g)$.

3: Create an empty heap $H$ of size $\# g$ and insert $(-1) f_{1}$ into $H$.

4: While the heap $H$ is not empty do

6 : Set $t:=0$.

7: Repeat

8: Extract $x:=H_{\max }$ from the heap and set $t:=t-x$.

9: Case $x=(-1) f_{i}$ and $i<\# f$ : Insert $(-1) f_{i+1}$ into $H$.

10: Case $x=g_{i} q_{j}$ and $j<\# q$ : Insert $g_{i} q_{j+1}$ into $H$.

11: Case $x=g_{i} q_{j}$ and $j=\# q$ : Set $s:=s+1(s=i)$.

12: Until $H$ is empty or $L M(t) \neq L M\left(H_{\max }\right)$.

13: If $t \neq 0$ and $L T(g) \mid t$ then

14: Copy $t / L T(g)$ onto the end of $q$.

15: For $i=2,3, \ldots, s$ compute $g_{i} \times(t / L T(g))$ and insert it into $H$.

16 : Set $s:=1$.

17: Else if $t \neq 0$ copy $t$ onto the end of $r$.

18: Output $(q, r)$.

Theorem 1. The divisor heap algorithm divides $f$ by $g$ producing the quotient $q$ and remainder $r$ using $O((\# f+\# q \# g) \log \# g)$ monomial comparisons and using storage for $O(\# g+\# q+\# r)$ terms.

Proof. We show that at Step $4,|H|+s-1=\# g$ if some $(-1) f_{i} \in H$ or $|H|+s=\# g$ otherwise. The first time Step 4 is executed, $|H|=1, s=\# g$, and $(-1) f_{1}$ is in the heap, so the loop invariant holds. Steps 7-11 extract a term from $H$ and either replace it or increment $s$, unless it was the last term of $f$. Step 15 inserts $s-1$ terms into $H$ and sets $s:=1$, maintaining the invariant.

Then $|H| \leq \# g$ since $s \geq 1$. Therefore the storage required is at most \#g terms in the heap plus the terms of $q$ and $r$. It should be clear that the algorithm adds terms of $f$, subtracts terms of each $g_{i} q$, and uses $L T(g)$ to cancel terms if possible, otherwise moving them to $r$, so that $f=q g+r$. Since we pass $\# f+\# q(\# g-1)$ terms through a heap of size $|H| \leq \# g$, the number of monomial comparisons is $O((\# f+\# q \# g) \log \# g)$. 


\subsection{Heap Optimizations}

We present two optimizations that are necessary to reproduce our results. The first is to implement the heap carefully. Many people are only aware of a bad algorithm for extracting the largest element from a heap, so we present a classical algorithm that is roughly twice as fast on average. As LaMarca and Ladner (9) observe, about $90 \%$ of the time is spent extracting elements from the heap so the resulting speedup is almost a factor of two.

We store the heap in a global dynamic array $H$, with the convention that $H[0]$ is the largest element and the children of $H[i]$ are $H[2 i+1]$ and $H[2 i+2]$.

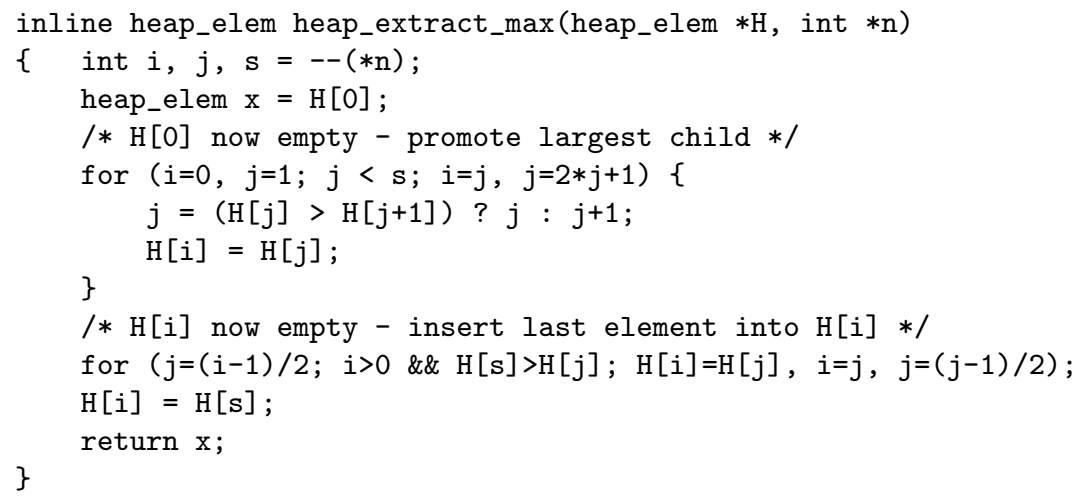

The extraction algorithm promotes the largest child into the empty space at a cost of one comparison per level of the heap $H$. Then it inserts the last element of the heap into the empty slot on the lowest level. However, since the last element was already a leaf, we do not expect it to travel very far up the heap. The number of comparisons required is $\log _{2}(n)+O(1)$ on average.

Compare this with the more commonly known algorithm for shrinking a heap, which moves the last element to the top and, at a cost of two comparisons per level (to find the maximum child and compare with it), sifts it down the heap. Since the last element was already a leaf it is likely to go all the way back down to the bottom, requiring $2 \log _{2}(n)$ comparisons on average.

Our second optimization improves performance when multiple terms are extracted from the heap. It is also necessary to obtain $O(n m)$ comparisons in the totally dense case. We insert and extract batches of terms instead of extracting a term and immediately inserting its successor. This requires a queue to store the extracted terms, however we can partition the heap to store this queue in place, as in heapsort. At the end of each iteration, we insert the successors of all of the extracted terms at once. As LaMarca notes (9), this strategy also produces favorable caching effects.

\subsection{Chaining terms with equal monomials}

Our next improvement chains heap elements with equal monomials to reduce the number of comparisons. Johnson (8) also experimented with this idea, however 
our scheme is simpler and we will show that multiplication and division of dense polynomials does $O(n m)$ comparisons.

We chain elements only as they are inserted into the heap, using an additional pointer in the structure that points to $f_{i}$ and $g_{j}$. In our implementation the pointers to $f_{i}$ and $g_{j}$ are not stored in the heap, but in a secondary structure that is accessed only when terms are inserted or extracted. Heap elements store a pointer to this structure and a pointer to the monomial product used for comparisons. The overhead of chaining elements in this way is negligible. The algorithms must be modified to check for chains and to extract all the elements of a chain without doing any monomial comparisons.

One final optimization is needed for multiplication. When multiplying $f g$, we must start with $f_{1} g_{1}$ in the heap and insert each $f_{i} g_{1}$ only after $f_{i-1} g_{1}$ has been extracted from the heap. This leads to the following results.

Lemma 1. Let $f$ and $g$ be dense univariate polynomials with $n$ and $m$ terms, respectively. A heap multiplication $f g$ with chaining does $n m-n-m+1$ comparisons.

Proof. We prove a loop invariant: at the beginning of each iteration the heap contains exactly one element or chain. This is true initially since the only element is $f_{1} g_{1}$. Each iteration removes the chain without doing a comparison, producing an empty heap. When we insert the successor terms into the heap all of the monomials are equal because the problem is dense, so all of the terms are chained together at the top of the heap. There are $n m$ terms and $n+m-1$ unique monomials. The first term with each monomial is inserted for free while the rest use one comparison each to chain. The total number of comparisons is thus $n m-n-m+1$.

Lemma 2. Let $q$ and $g$ be dense univariate polynomials with $n$ and $m$ terms and let $f=q g$. Then a quotient heap division $f \div g$ with chaining does $n m-n$ comparisons.

Proof. We use the same loop invariant: the heap contains exactly one element or chain, which is initially $f_{1}$. Each iteration extracts the terms of this chain, adding their coefficients without a comparison, producing an empty heap. If the term is not zero, a new term of the quotient $q_{i}$ is computed and the monomial of $q_{i} g_{1}$ equal to the monomial of the extracted terms. When we insert its successor $q_{i} g_{2}$ and the successors of all the other terms their monomials are all equal because the problem is dense, and all of the terms are chained together at the top of the heap. If each of the $n+m-1$ monomials of $f$ is inserted first without any comparisons, the remaining $n(m-1)$ terms of $-q(g-L T(g))$ will be chained using one comparison each.

Remark: The divisor heap algorithm can also be modified to do $\mathrm{nm}$ comparisons in the dense univariate case. Each term $q_{j}$ of the quotient should insert only $g_{2} q_{j}$ if it is not in the heap, and each $g_{i+1} q_{j}$ should be inserted only after $g_{i} q_{j}$ is extracted from the heap. We have not yet implemented this modification. 


\section{Benchmarks}

\subsection{The number of monomial comparisons}

Our first benchmark (see Table 1 and Table 2) is due to Johnson (8). We multiply and divide sparse univariate polynomials and report the number of comparisons divided by the total number of terms that are merged. Recall that for a sparse multiplication $f g$ this is $(\# f)(\# g)$ and for a sparse division $f=q g$ this is $\# f+\# q(\# g-1)$. A "structure parameter" $S$ is used to randomly generate polynomials $f=a_{0}+a_{1} x^{e_{1}}+a_{2} x^{e_{2}}+\cdots+a_{k} x^{e_{k}}$ with the difference between the exponents satisfying $1 \leq e_{i+1}-e_{i} \leq S$.

For each problem we generate $f$ and $g$ with $n$ and $m$ terms respectively, multiply $p=f g$ and divide $p / g$. For multiplication we test both chained and unchained heaps, and for division we test the "quotient heap" and the "divisor heap" algorithms.

Table 1. Multiplication $f g$ and the number of comparisons divided by $(\# f)(\# g)$.

\begin{tabular}{|rrr|rrrr|}
\hline $\mathrm{S}$ & $\#(f g)$ & $\# f, \# g$ & unchained heap & chained heap & geobuckets & direct merge \\
\hline 1 & 199 & 100 & 6.138 & .980 & 1.114 & 1.475 \\
& 1999 & 1000 & 9.329 & .998 & 1.027 & 1.497 \\
\hline 10 & 1025 & 100 & 8.339 & 5.970 & 2.905 & 7.239 \\
& 10747 & 1000 & 11.717 & 8.478 & 3.065 & 8.025 \\
\hline 100 & 5728 & 100 & 8.671 & 8.282 & 4.690 & 32.893 \\
& 97051 & 1000 & 11.879 & 11.334 & 5.798 & 69.191 \\
\hline 1000 & 9364 & 100 & 8.805 & 8.748 & 5.274 & 48.073 \\
& 566984 & 1000 & 11.925 & 11.852 & 7.511 & 324.135 \\
\hline
\end{tabular}

Table 2. Division $f g \div g$ and the number of comparisons divided by $\#(f g)+\# f(\# g-1)$.

\begin{tabular}{|rrrr|rrrr|}
\hline $\mathrm{S}$ & $\#(f g)$ & $\# f$ & $\# g$ & quotient heap & divisor heap & geobuckets & direct merge \\
\hline 1 & 199 & 100 & 100 & .980 & 2.627 & .980 & .980 \\
& 1099 & 100 & 1000 & .989 & 7.622 & .989 & .989 \\
& 1099 & 1000 & 100 & .989 & 1.155 & .989 & .999 \\
& 1999 & 1000 & 1000 & .998 & 4.170 & .998 & .998 \\
\hline 10 & 1025 & 100 & 100 & 5.692 & 6.480 & 2.647 & 4.300 \\
& 5856 & 100 & 1000 & 6.493 & 8.244 & 2.738 & 4.872 \\
& 5949 & 1000 & 100 & 6.503 & 7.825 & 2.748 & 4.934 \\
& 11162 & 1000 & 1000 & 8.646 & 9.124 & 2.916 & 5.473 \\
\hline 100 & 5725 & 100 & 100 & 7.106 & 7.580 & 3.945 & 14.502 \\
& 44725 & 100 & 1000 & 7.884 & 10.594 & 3.954 & 19.381 \\
& 45358 & 1000 & 100 & 7.696 & 7.938 & 4.405 & 18.231 \\
& 96443 & 1000 & 1000 & 10.898 & 11.438 & 5.471 & 42.262 \\
\hline 1000 & 9403 & 100 & 100 & 7.116 & 7.522 & 3.992 & 17.307 \\
& 90884 & 100 & 1000 & 7.682 & 10.608 & 4.253 & 23.978 \\
& 91141 & 1000 & 100 & 7.658 & 7.747 & 4.596 & 22.736 \\
& 571388 & 1000 & 1000 & 10.563 & 11.056 & 6.574 & 142.095 \\
\hline
\end{tabular}

We make a couple of remarks concerning tables 1 and 2. First it should be clear that our implementation of the divisor heap algorithm is not fully optimized. As discussed at the end of Section 3 we should delay inserting products $g_{i} q_{j}$ into the heap until after the previous product $g_{i-1} q_{j}$ is extracted from the heap. This is needed to obtain $O(n m)$ comparisons in the dense case $(S=1)$. 
Second, it is interesting to see that geobuckets do roughly half the number of comparisons as the heap algorithms in the sparse case, and this ratio improves as the problems become more dense. We tried some improvements to the heap algorithms such as chaining elements while shrinking the heap, however these changes tended to decrease the real world performance of the algorithms.

\subsection{7 variable cofactor problem}

Our next benchmark (see Table 3) simulates a GCD problem. A large sparse polynomial is divided by one of its factors (the GCD) to compute the cofactor. To generate this example we constructed four polynomials $\left\{f_{1}, f_{2}, f_{3}, f_{4}\right\}$ and divided their product $p=f_{1} f_{2} f_{3} f_{4}$ by $f_{1}, f_{1} f_{2}$, and $f_{1} f_{2} f_{3}$ over $\mathbb{Z}_{32003}$ using graded lexicographic order. The polynomials have $\# f_{i}=50$ and $\operatorname{deg}\left(f_{i}\right)=10$.

The computations were performed on an AMD Opteron $2542.8 \mathrm{GHz}$ with $8 \mathrm{~GB}$ of $400 \mathrm{MHz}$ RAM and $1 \mathrm{MB}$ of L2 cache running 64-bit Red Hat Enterprise Linux 5 with a 2.6 .18 kernel.

Table 3. Sparse multiplications and divisions in 7 variables over $\mathbb{Z}_{32003}$ using graded lex order with $\{1,2,4,8\}$ exponents packed into each 64 -bit word. $\# f_{i}=50, \operatorname{deg}\left(f_{i}\right)=10$, $\#\left(f_{1} f_{2}\right)=2492, \#\left(f_{3} f_{4}\right)=2491, \#\left(f_{1} f_{2} f_{3}\right)=121903, \#\left(f_{1} f_{2} f_{3} f_{4}\right)=4523085$.

\begin{tabular}{|c|c|c|c|c|c|c|}
\hline \multicolumn{7}{|c|}{$\begin{array}{l}\left(f_{1} f_{2}\right) \times\left(f_{3} f_{4}\right) \\
\text { expon/wd size of result }\end{array}$} \\
\hline 1 & $310.57 \mathrm{MB}$ & $2.630 \mathrm{~s} \quad(0.38 \mathrm{MB})$ & $7.720 \mathrm{~s}$ & (994 MB) & $332.230 \mathrm{~s}$ & (371 MB) \\
\hline 2 & $172.54 \mathrm{MB}$ & $(0.31 \mathrm{MB})$ & $4.230 \mathrm{~s}$ & $(552 \mathrm{MB})$ & $185.780 \mathrm{~s}$ & (206 MB) \\
\hline 4 & $103.52 \mathrm{MB}$ & $(0.27 \mathrm{MB})$ & $2.550 \mathrm{~s}$ & $(331 \mathrm{MB})$ & $111.960 \mathrm{~s}$ & (124 MB) \\
\hline 8 & $69.01 \mathrm{MB}$ & $(0.25 \mathrm{MB})$ & $1.760 \mathrm{~s}$ & $(221 \mathrm{MB})$ & $75.560 \mathrm{~s}$ & $(83 \mathrm{MB})$ \\
\hline \multicolumn{7}{|c|}{$f_{1} \times\left(f_{2} f_{3} f_{4}\right)$} \\
\hline expon/wd & size of result & chained heap & \multicolumn{2}{|c|}{ geobuckets } & \multicolumn{2}{|c|}{ direct merge } \\
\hline 1 & $310.57 \mathrm{MB}$ & (0.07 MB) & $4.770 \mathrm{~s}$ & (1143 MB) & $8.070 \mathrm{~s}$ & (483 MB) \\
\hline 2 & $172.54 \mathrm{MB}$ & $(0.06 \mathrm{MB})$ & $2.660 \mathrm{~s}$ & $(635 \mathrm{MB})$ & $4.500 \mathrm{~s}$ & (216 MB) \\
\hline 4 & $103.52 \mathrm{MB}$ & $(0.06 \mathrm{MB})$ & $1.690 \mathrm{~s}$ & $(381 \mathrm{MB})$ & $2.800 \mathrm{~s}$ & (161 MB) \\
\hline 8 & $69.01 \mathrm{MB}$ & $(0.06 \mathrm{MB})$ & $1.230 \mathrm{~s}$ & $(254 \mathrm{MB})$ & $1.910 \mathrm{~s}$ & (107 MB) \\
\hline
\end{tabular}

$\left(f_{1} f_{2} f_{3} f_{4}\right) /\left(f_{1} f_{2} f_{3}\right)$
\begin{tabular}{|c|cc|cr|rr|rr|}
\hline $\mathrm{x}$ & \multicolumn{2}{|c|}{ quotient heap } & \multicolumn{2}{c|}{ divisor heap } & \multicolumn{2}{c|}{ geobuckets } & \multicolumn{2}{c|}{ direct merge } \\
\hline 1 & $2.000 \mathrm{~s}$ & $(0.13 \mathrm{MB})$ & $8.820 \mathrm{~s}$ & $(18.6 \mathrm{MB})$ & $5.190 \mathrm{~s}$ & $(1793 \mathrm{MB})$ & $7.530 \mathrm{~s}$ & $(944 \mathrm{MB})$ \\
2 & $1.450 \mathrm{~s}$ & $(0.13 \mathrm{MB})$ & $6.570 \mathrm{~s}$ & $(14.9 \mathrm{MB})$ & $2.960 \mathrm{~s}$ & $(996 \mathrm{MB})$ & $4.250 \mathrm{~s}$ & $(524 \mathrm{MB})$ \\
4 & $1.250 \mathrm{~s}$ & $(0.10 \mathrm{MB})$ & $5.270 \mathrm{~s}$ & $(13.0 \mathrm{MB})$ & $1.950 \mathrm{~s}$ & $(598 \mathrm{MB})$ & $2.610 \mathrm{~s}$ & $(315 \mathrm{MB})$ \\
8 & $1.060 \mathrm{~s}$ & $(0.10 \mathrm{MB})$ & $4.530 \mathrm{~s}$ & $(12.1 \mathrm{MB})$ & $1.500 \mathrm{~s}$ & $(398 \mathrm{MB})$ & $1.770 \mathrm{~s}$ & $(210 \mathrm{MB})$ \\
\hline
\end{tabular}

$\left(f_{1} f_{2} f_{3} f_{4}\right) /\left(f_{1} f_{2}\right)$
\begin{tabular}{|c|cr|rr|rr|rr|}
\hline $\mathrm{x}$ & \multicolumn{2}{|c|}{ quotient heap } & \multicolumn{2}{c|}{ divisor heap } & \multicolumn{2}{c|}{ geobuckets } & \multicolumn{2}{c|}{ direct merge } \\
\hline 1 & $3.270 \mathrm{~s}$ & $(0.72 \mathrm{MB})$ & $3.380 \mathrm{~s}$ & $(0.30 \mathrm{MB})$ & $8.020 \mathrm{~s}$ & $(1461 \mathrm{MB})$ & $330.730 \mathrm{~s}$ & $(932 \mathrm{MB})$ \\
2 & $2.290 \mathrm{~s}$ & $(0.65 \mathrm{MB})$ & $2.430 \mathrm{~s}$ & $(0.31 \mathrm{MB})$ & $4.460 \mathrm{~s}$ & $(812 \mathrm{MB})$ & $183.060 \mathrm{~s}$ & $(518 \mathrm{MB})$ \\
4 & $1.840 \mathrm{~s}$ & $(0.62 \mathrm{MB})$ & $1.930 \mathrm{~s}$ & $(0.27 \mathrm{MB})$ & $2.760 \mathrm{~s}$ & $(487 \mathrm{MB})$ & $110.290 \mathrm{~s}$ & $(311 \mathrm{MB})$ \\
8 & $1.520 \mathrm{~s}$ & $(0.60 \mathrm{MB})$ & $1.620 \mathrm{~s}$ & $(0.25 \mathrm{MB})$ & $2.040 \mathrm{~s}$ & $(321 \mathrm{MB})$ & $74.540 \mathrm{~s}$ & $(207 \mathrm{MB})$ \\
\hline
\end{tabular}

\begin{tabular}{|c|c|c|c|c|c|c|c|}
\hline $\mathrm{xt}$ & \multicolumn{7}{|c|}{$\left(f_{1} f_{2} f_{3} f_{4}\right) / f_{1}$} \\
\hline & & & & & & & \\
\hline \begin{tabular}{|l|}
1 \\
\end{tabular} & $8.010 \mathrm{~s}$ & $(28.46 \mathrm{MB})$ & $1.990 \mathrm{~s}$ & $(0.07 \mathrm{MB})$ & $8.320 \mathrm{~s}$ & $(1371 \mathrm{MB})$ & - \\
\hline 2 & $5.900 \mathrm{~s}$ & $(25.69 \mathrm{MB})$ & $1.480 \mathrm{~s}$ & $(0.06 \mathrm{MB})$ & $4.640 \mathrm{~s}$ & $(762 \mathrm{MB})$ & - \\
\hline 4 & $4.750 \mathrm{~s}$ & $(24.29 \mathrm{MB})$ & $1.240 \mathrm{~s}$ & $(0.06 \mathrm{MB})$ & $2.890 \mathrm{~s}$ & $(457 \mathrm{MB})$ & - \\
\hline 8 & $3.970 \mathrm{~s}$ & $(23.60 \mathrm{MB})$ & $1.080 \mathrm{~s}$ & $(0.06 \mathrm{MB})$ & $2.210 \mathrm{~s}$ & (305 MB) & $3526.750 \mathrm{~s}$ \\
\hline
\end{tabular}


We report times and memory for $\{1,2,4,8\}$ exponents per 64 -bit word. For multiplications we subtracted the size of the product from the memory totals for the geobucket and merge algorithms, and for divisions we did not include memory for the quotient. For heap algorithms we report the size of the heap and products. Thus we report the memory overhead of the algorithms, not the total memory used. For divisions the largest quotient $\left(f_{2} f_{3} f_{4}\right)$ is at most 8.3 MB.

The heap algorithms performed very well on this example despite their higher cost in monomial comparisons. We attribute this to the fact that their working memory (the heap of pointers and the monomial products) fits in the L2 cache, whereas geobuckets and direct merging work mostly in RAM, which is 7 times slower than the processor.

Also note the effect of packing exponents. The performance of merging and geobuckets is practically linear in the size of the terms, which is $9,5,3$, or 2 words with the coefficient. The heap algorithms do not benefit as much, but the improvement is worthwhile. Going from 64-bit (1 exponent per word) to 16-bit (4 exponents per word) exponents places only modest restrictions on the total degree and improves performance by $40 \%$.

\subsection{The effect of faster RAM and a larger L2 cache}

In the previous benchmark the performance of geobuckets was constrained by the speed of the RAM and the size of the L2 cache. We thought that geobuckets should outperform the heap algorithms under different conditions, because they typically do fewer monomial comparisons.

Table 4. Sparse multiplications and divisions in 4 variables over $\mathbb{Z}_{32003}$. Lexicographic order was used with 32 -bit words. Each $f_{i}$ has degree 30 in each variable. $\# f_{1}=96$, $\# f_{2}=93, \# f_{3}=93, \#\left(f_{1} f_{2}\right)=8922, \#\left(f_{2} f_{3}\right)=8639, \#\left(f_{1} f_{2} f_{3}\right)=795357$.

\begin{tabular}{|c|c|c|c|c|c|}
\hline \multicolumn{6}{|c|}{$f_{1} \times\left(f_{2} f_{3}\right)$} \\
\hline & 1 & $15.17 \mathrm{MB}$ & $0.200 \mathrm{~s} \quad(0.03 \mathrm{MB})$ & $0.210 \mathrm{~s} \quad(55.74 \mathrm{MB})$ & $0.650 \mathrm{~s} \quad(23.21 \mathrm{MB})$ \\
\hline & 2 & $9.10 \mathrm{MB}$ & $0.150 \mathrm{~s} \quad(0.03 \mathrm{MB})$ & $(33.44 \mathrm{MB})$ & $0.470 \mathrm{~s} \quad(13.92 \mathrm{MB})$ \\
\hline & 4 & $6.07 \mathrm{MB}$ & $(0.03 \mathrm{MB})$ & $(22.30 \mathrm{MB})$ & $(9.28 \mathrm{MB})$ \\
\hline \multicolumn{6}{|c|}{$\left(f_{1} f_{2} f_{3}\right) /\left(f_{1} f_{2}\right)$} \\
\hline $\mathrm{x} / \mathrm{w}$ & \multicolumn{2}{|c|}{ quotient heap } & divisor heap & geobuckets & direct merge \\
\hline 1 & $0.260 \mathrm{~s}$ & $(0.06 \mathrm{MB})$ & $0.460 \mathrm{~s} \quad(0.55 \mathrm{MB})$ & $(70.91 \mathrm{MB})$ & $0.600 \mathrm{~s} \quad(38.38 \mathrm{MB})$ \\
\hline 2 & $0.210 \mathrm{~s}$ & $(0.05 \mathrm{MB})$ & (0.48 MB) & $(37.38 \mathrm{MB})$ & $0.440 \mathrm{~s} \quad(27.46 \mathrm{MB})$ \\
\hline 4 & $0.170 \mathrm{~s}$ & $(0.05 \mathrm{MB})$ & $(0.45 \mathrm{MB})$ & $(22.36 \mathrm{MB})$ & (18.30 MB) \\
\hline \multicolumn{6}{|c|}{$\left(f_{1} f_{2} f_{3}\right) / f_{1}$} \\
\hline $\mathrm{x} / \mathrm{w}$ & \multicolumn{2}{|c|}{ quotient heap } & divisor heap & geobuckets & direct merge \\
\hline 1 & $0.430 \mathrm{~s}$ & $(0.53 \mathrm{MB})$ & $(0.03 \mathrm{MB})$ & $(55.90 \mathrm{MB})$ & $(45.52 \mathrm{MB})$ \\
\hline 2 & $0.350 \mathrm{~s}$ & $(0.47 \mathrm{MB})$ & $(0.03 \mathrm{MB})$ & $(33.54 \mathrm{MB})$ & $28.790 \mathrm{~s} \quad(27.30 \mathrm{MB})$ \\
\hline 4 & $0.280 \mathrm{~s}$ & $(0.43 \mathrm{MB})$ & $(0.03 \mathrm{MB})$ & $(22.36 \mathrm{MB})$ & $(18.20 \mathrm{MB})$ \\
\hline
\end{tabular}

Our third benchmark (see Table 4) is a smaller problem similar to the previous one. We created three random polynomials $\left\{f_{1}, f_{2}, f_{3}\right\}$ and divided their product by $f_{1}$ and $f_{2} f_{3}$. This test was run on a $2.4 \mathrm{GHz}$ Intel E6600 Core 2 Duo with $2 \mathrm{~GB}$ of $666 \mathrm{MHz}$ RAM, $4 \mathrm{MB}$ of L2 cache, and 32-bit words running 
Fedora Core 6. Thus RAM is now only 3.6 times slower than the CPU and the number of words in the L2 cache has increased by a factor of eight.

Table 4 shows that geobuckets are competitive with heap algorithms if they work in the L2 cache. The times include memory allocation, so in practice if the geobucket is reused it may be faster than a quotient heap on sparse problems, with an additional advantage on dense problems (see tables 1 and 2). However when the quotient is large, the divisor heap's lower complexity easily wins.

\subsection{Algebraic extensions}

Our final benchmark (see Table 5) is a large division with algebraic extensions. We constructed four random polynomials $\left\{f_{1}, f_{2}, f_{3}, f_{4}\right\}$ in $\mathbb{Z}_{32003}[x, y, z, \alpha, \beta, s, t]$ with $\operatorname{deg}\left(f_{i}\right)=10$ and $L T\left(f_{i}\right)=x^{10}$. We used lexicographic order with $x>y>$ $z>\alpha>\beta>s>t$ with the extensions $\alpha^{2}-3=0$ and $\beta^{2}+s t-1=0$. Thus we are effectively computing with polynomials in $\{x, y, z\}$ with coefficients in $\mathbb{Z}_{32003}[\alpha, \beta, s, t] /\left\langle\alpha^{2}-3, \beta^{2}+s t-1\right\rangle$.

We report the times to multiply $\left(f_{1} f_{2}\right) \times\left(f_{3} f_{4}\right)$ and $f_{4} \times\left(f_{1} f_{2} f_{3}\right)$ and reduce the product mod $\left\{\alpha^{2}-3, \beta^{2}+s t-1\right\}$. Next we divide the reduced product by $f_{1},\left(f_{1} f_{2}\right)$, and $\left(f_{1} f_{2} f_{3}\right)$ mod $\left\{\alpha^{2}-3, \beta^{2}+s t-1\right\}$ and reduce the quotients $\bmod \left\{\alpha^{2}-3, \beta^{2}+s t-1\right\}$. The divisors in each case are already reduced mod $\left\{\alpha^{2}-3, \beta^{2}+s t-1\right\}$.

We performed the test on a $3 \mathrm{GHz}$ Intel Xeon 5160 with $16 \mathrm{~GB}$ of 666 $\mathrm{MHz}$ RAM and $4 \mathrm{MB}$ of L2 cache running 64-bit Red Hat Enterprise Linux 5 . Memory numbers are reported differently since the heap algorithms must store the quotients of $\left\{\alpha^{2}-3, \beta^{2}+s t-1\right\}$ which are large, whereas geobuckets discards them. We report the total memory allocated by each routine, including reallocations to enlarge the geobucket and speculative allocations of quotients by the heap algorithms. We pack all seven exponents into one 64-bit word. The results with less packing are consistent with our previous benchmarks.

Table 5. Sparse multiplications and divisions with algebraic extensions. Lexicographic order was used with 7 exponents per 64-bit word. We include the times, the number of monomial comparisons (upper right), and the total memory allocated. $\# f_{1}=106$, $\# f_{2}=96, \# f_{3}=105, \# f_{4}=98, \#\left(f_{1} f_{2}\right)=8934, \#\left(f_{3} f_{4}\right)=8982, \#\left(f_{1} f_{2} f_{3}\right)=$ 256685, \# $\left(f_{1} f_{2} f_{3} f_{4}\right)=1663235$.

\begin{tabular}{|l|rl|rl|rl|}
\hline & \multicolumn{2}{|c|}{ quotient heap } & \multicolumn{2}{c|}{ divisor heap } & \multicolumn{2}{c|}{ geobuckets } \\
\hline$p=\left(f_{1} f_{2}\right)\left(f_{3} f_{4}\right)$ & $11.080 \mathrm{~s}$ & $9.713 \times 10^{8}$ & $11.100 \mathrm{~s}$ & $9.267 \times 10^{8}$ & $8.510 \mathrm{~s}$ & $4.218 \times 10^{8}$ \\
reduce product & $0.700 \mathrm{~s}$ & $458.75 \mathrm{MB}$ & $0.300 \mathrm{~s}$ & $166.73 \mathrm{MB}$ & $0.610 \mathrm{~s}$ & $646.54 \mathrm{MB}$ \\
\hline$p=f_{4}\left(f_{1} f_{2} f_{3}\right)$ & $1.690 \mathrm{~s}$ & $1.966 \times 10^{8}$ & $1.680 \mathrm{~s}$ & $1.546 \times 10^{8}$ & $2.130 \mathrm{~s}$ & $8.184 \times 10^{7}$ \\
reduce product & $0.670 \mathrm{~s}$ & $446.07 \mathrm{MB}$ & $0.300 \mathrm{~s}$ & $163.12 \mathrm{MB}$ & $0.560 \mathrm{~s}$ & $642.30 \mathrm{MB}$ \\
\hline$p /\left(f_{1} f_{2} f_{3}\right)$ & $3.060 \mathrm{~s}$ & $2.862 \times 10^{8}$ & $11.910 \mathrm{~s}$ & $6.949 \times 10^{8}$ & $3.360 \mathrm{~s}$ & $1.218 \times 10^{8}$ \\
reduce quotient & $0.000 \mathrm{~s}$ & $208.02 \mathrm{MB}$ & $0.000 \mathrm{~s}$ & $64.34 \mathrm{MB}$ & $0.000 \mathrm{~s}$ & $479.98 \mathrm{MB}$ \\
\hline$p /\left(f_{1} f_{2}\right)$ & $51.430 \mathrm{~s}$ & $4.097 \times 10^{9}$ & $35.040 \mathrm{~s}$ & $2.860 \times 10^{9}$ & $35.520 \mathrm{~s}$ & $1.732 \times 10^{9}$ \\
reduce quotient & $0.010 \mathrm{~s}$ & $733.72 \mathrm{MB}$ & $0.010 \mathrm{~s}$ & $81.45 \mathrm{MB}$ & $0.010 \mathrm{~s}$ & $1205.19 \mathrm{MB}$ \\
\hline$p / f_{1}$ & $49.790 \mathrm{~s}$ & $2.005 \times 10^{9}$ & $5.980 \mathrm{~s}$ & $4.616 \times 10^{8}$ & $13.140 \mathrm{~s}$ & $9.100 \times 10^{8}$ \\
reduce quotient & $0.190 \mathrm{~s}$ & $752.61 \mathrm{MB}$ & $0.080 \mathrm{~s}$ & $113.25 \mathrm{MB}$ & $0.180 \mathrm{~s}$ & $1038.96 \mathrm{MB}$ \\
\hline
\end{tabular}


The divisor heap algorithm performs well on this example (and the quotient heap algorithm poorly) because $\left\{\alpha^{2}-3, \beta^{2}+s t-1\right\}$ are small divisors with large quotients, i.e., they are frequently used to reduce terms during the division. The time and space requirements of the divisor heap algorithm scale linearly with the total number of reduction steps, so we expect it to be especially useful for divisions in the presence of algebraic extensions.

Geobuckets also perform well on this benchmark. Their overall memory usage is low because they do not need to store all of the quotients and the number of monomial comparisons they do is very competitive. However, performance is not dictated entirely by monomial comparisons. Consider the fourth benchmark $p /\left(f_{1} f_{2}\right)$, where geobuckets do half the number of monomial comparisons as a divisor heap only to finish in the same amount of time.

The performance of geobuckets suffers because they access a large amount of memory randomly, and this decreases the effectiveness of the cache. Imagine what happens when $\beta^{2}+s t-1$ is used to reduce one million terms in a row. Geobuckets will merge multiples of this polynomial into the smallest bucket $10^{6}$ times, interspersed with 500,000 merges into the second bucket, 250,000 merges into the third, and so on. When a large bucket is merged the smaller buckets are evicted from the cache, producing cache misses the next time those buckets are accessed. If the problem is sufficiently large or the L2 cache is small, this will happen frequently.

By contrast, the divisor heap algorithm will do two simultaneous passes over the quotient of $\beta^{2}+s t-1$ while randomly accessing a heap with three elements, two monomial products, and the terms of the divisor. This is a tiny amount of memory, so almost all of the cache is used to load terms from the quotient, and very few cache misses will occur.

\section{Conclusions and Future Work}

We have shown how a heap of pointers can be very efficient for sparse polynomial division and multiplication. This performance is primarily due to the very low memory requirements of the algorithms and their cache-friendly design. We have also presented a new division algorithm that scales linearly with the size of the quotient(s) by using a heap the size of the divisor(s). This algorithm should have many applications for polynomial computations with algebraic extensions.

In the future we plan to combine the quotient and divisor heap algorithms to produce a division algorithm which is $O(n m \log (\min (n, m)))$, which we believe is optimal. We also plan to implement versions of the heap algorithms that use GMP for large integer arithmetic, and we are experimentally trying to parallelize the heap algorithms as well. 


\section{Bibliography}

[1] Olaf Bachmann and Hans Schönemann. Monomial representations for Gröbner bases computations. Proceedings of ISSAC 1998, ACM Press (1998) 309-316.

[2] W. S. Brown. Altran Users Manual. 4th edition. Bell Labs, Murray Hill, N.J., 1977.

[3] David Cox, John Little, Donal O'Shea. Ideals, Varieties and Algorithms: An Introduction to Computational Algebraic Geometry and Commutative Algebra. Springer Verlag, 1992.

[4] K. O. Geddes, S. R. Czapor, and G. Labahn. Algorithms for Computer Algebra, Kluwer Academic, 1992.

[5] D. R. Grayson and M. E. Stillman. MAcAulay 2, a software system for research in algebraic geometry. Available at http://www . math. uiuc . edu/Macaulay2/

[6] G.-M. Greuel, G. Pfister, and H. Schönemann. Singular 3.0. A Computer Algebra System for Polynomial Computations. Centre for Computer Algebra, University of Kaiserslautern (2005). http: //www . singular.uni-kl.de.

[7] Richard Jenks, Robert Sutor and Scott Morrison. AXIOM: The Scientific Computation System Springer-Verlag, 1992.

[8] Stephen C. Johnson. Sparse polynomial arithmetic. ACM SIGSAM Bulletin, Volume 8, Issue 3 (1974) 63-71.

[9] Anthony LaMarca, Richard Ladner. The Influence of Caches on the Performance of Heaps. J. Experimental Algorithms 1, Article 4, 1996.

[10] M. Monagan, K. Geddes, K. Heal, G. Labahn, S. Vorkoetter, J. McCarron, and P. DeMarco. Maple 10 Introductory Programming Guide Maplesoft, ISBN 1-894511-76, 2005.

[11] David Stoutemyer. Which Polynomial Representation is Best? Proceedings of the 1984 Macsyma Users Conference Schenectedy, N.Y., (1984) 221-244.

[12] Thomas Yan. The Geobucket Data Structure for Polynomials. J. Symb. Comput. 25 (1998) 285-293. 